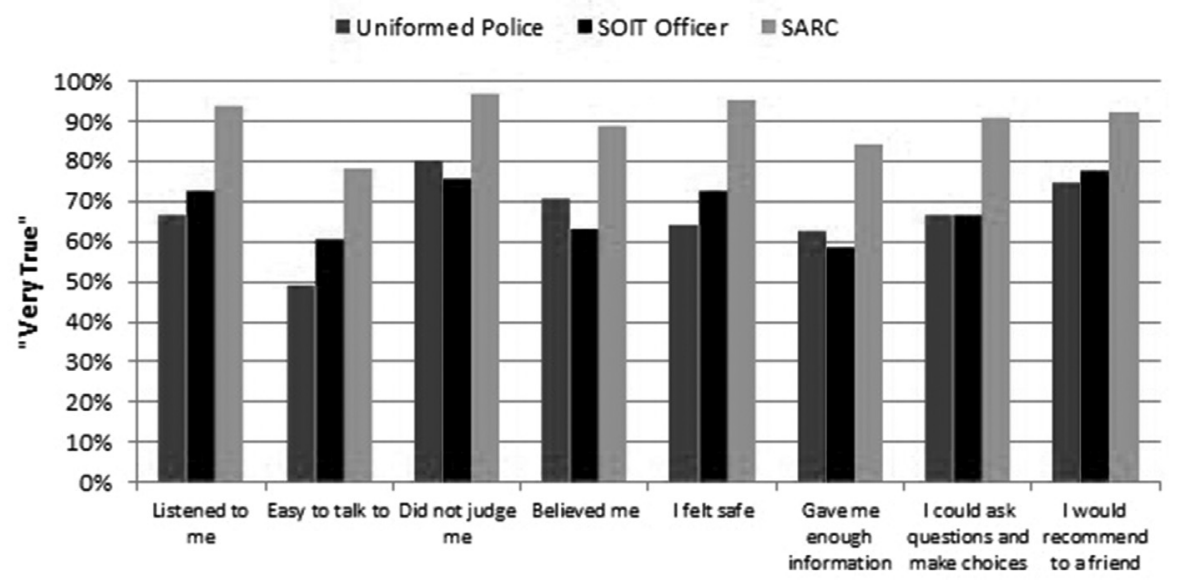

Abstract G147 Figure 1 Adolescent experiences of care following sexual assault

for Health Improvement Experience of Service Questionnaire (CHI-ESQ).

Results 30\% consented to take part. Data were analysed for the first 64 study participants, evaluating care received from uniformed police $(\mathrm{n}=51)$, specialist sexual offences investigative techniques (SOIT) officers $(n=58)$ and clinical staff at the SARCs (doctors and crisis workers, $\mathrm{n}=64$ ). $97 \%$ were police referrals and $3 \%$, self-referrals. $67 \%$ had experienced vaginal rape, $32 \%$, oral rape and $12 \%$, anal rape. $90 \%$ underwent a forensic medical examination.

Commonly held fears before accessing care were that they would be judged, disbelieved, 'blamed' or 'called a liar'. Many participants were apprehensive that the forensic examination would be painful, embarrassing or reveal health problems such as infections.

As shown in Figure 1, care given by clinical staff at SARCs was consistently rated higher than police care. Only $49 \%$ found uniformed police easy to talk to and only $64 \%$ felt safe in their care. Only $63 \%$ felt believed by their SOIT officer and only $59 \%$ felt they had been given enough information (Figure 1).

Conclusions Many participants described positive experiences of systems of care for victims of sexual assault. However, variable experiences of care between services highlight the need for improvement in specific areas. Anxieties about being blamed or judged may prevent adolescents accessing services following sexual assault.

\section{G148 THE RELATIONSHIP BETWEEN PUBERTAL STATUS AND NEURAL ACTIVITY DURING RISKY DECISION-MAKING IN MALE ADOLESCENTS USING FMRI}

\footnotetext{
${ }^{1,2} \mathrm{AL}$ Goddings, ${ }^{1,2} \mathrm{~L}$ Menzies, ${ }^{3}$ I Dumontheil, ${ }^{2} \mathrm{E}$ Garrett, ${ }^{2} \mathrm{~S} J$ Blakemore, ${ }^{1} \mathrm{RM}$ Viner. ${ }^{1}$ Institute of Child Health, University College London, London, UK; ${ }^{2}$ Institute of Cognitive Neuroscience, University College London, London, UK; ${ }^{3}$ Department of Psychological Sciences, Birkbeck College, University of London, London, UK
}

\subsection{6/archdischild-2015-308599.145}

During adolescence, risk-taking emerges as an important behaviour. One prominent theory proposes that this increased risk-taking results from a dissociation in the maturational timing of the brain regions involved in reward processing and cognitive control. It is hypothesised that the regions involved in reward processing, particularly the limbic system, mature relatively early in adolescence, in tandem with pubertal maturation. In contrast, the cognitive control regions of the brain, found principally within the prefrontal cortex, are proposed to undergo protracted development throughout adolescence. The goal of this functional Magnetic Resonance Imaging (fMRI) study was to explore how developmental changes in brain function when performing a risk-taking task were related to puberty, independently of chronological age.

Fifty male participants aged 12-14 years underwent fMRI scanning whilst performing a risk-taking task (the BART task). Indicators of pubertal development were collected, including self-reported pubertal status using Tanner stage diagrams and salivary hormone levels for testosterone, oestradiol and dehydroepiandrosterone (DHEA). Participants were grouped by pubertal status into early-mid puberty ( $\leq$ Tanner Stage 3 in pubic hair and gonadal development; $\mathrm{n}=23$ ) and late-post puberty ( $\geq$ Tanner Stage 4 in either pubic hair or gonadal development; $n$ $=26)$.

General linear modelling was used to investigate whether there were differences in neural activity within the reward processing and cognitive control brain networks during the risk-taking task with pubertal development, as measured by Tanner stage puberty groups and hormonal levels. Oestradiol levels were positively correlated with neural activity in the medial prefrontal cortex and orbitofrontal cortex when deciding not to make a risky choice. Testosterone levels and puberty grouping (early-mid vs. late-post) were correlated with prefrontal cortex activation during the processing of outcomes of a risky decision.

This study highlights the complexity of the decision-making process, and the extensive network of brain regions involved. It suggests that various aspects of the process of deciding to make a risky decision are related to distinct elements of pubertal development.

\section{G149 THE MANAGEMENT OF EATING DISORDERS ON CHILDREN'S WARDS: TRIALS, TRIBULATIONS AND TRAINING}

${ }^{1}$ A Yeadon, ${ }^{2}$ Mannion. ${ }^{1}$ School of Paediatrics, Health Education Yorkshire and the Humber, UK; ${ }^{2}$ Children's Ward, York Teaching Hospital NHS Foundation Trust, York, UK

\subsection{6/archdischild-2015-308599.146}

Aims This series of studies considers the challenges faced when caring for young people with eating disorders on children's wards, suggesting approaches to improving quality of care. 
Methods This report brings together information from four sources, all referencing the standards defined by Junior MARSIPAN:

1. Audit of 12 admissions to Hospital A, assessing quality of medical care

2. Audit of 4 simultaneous admissions to Hospital B, considering pragmatic aspects of care

3. Review of a particularly challenging case admitted to Hospital A

4. Deanery-wide trainee survey (22 responses, ST4-8)

Results

1. At least half of young people displayed high risk clinical parameters, but assessment was frequently incomplete. Postural observations were rarely recorded.\%BMI was only documented in $17 \%$. Hydration status was considered in $8 \%$. However, most patients had an ECG, and monitoring for refeeding syndrome was adequate.

2. All four young people displayed multiple high risk medical features. Despite all experiencing symptoms for over 9 months, each was known to CAMHS for less than 2 months. Limited confidence in managing these patients was acknowledged amongst both medical and nursing staff. Particular challenges included adequate supervision, lack of specialist dietetic advice, and communication between paediatric and CAMHS teams.

3. This case involved a teenage girl admitted for nasogastric refeeding. Compliance was limited from the outset. Despite sedation, full time specialist nursing support and frequent restraint, feeds could not be safely administered and her condition deteriorated significantly. A specialist bed only became available after a 13 day admission.

4. Despite three-quarters having encountered at least one young person with an eating disorder in the last 2 years, only one in five had received any formal training. $82 \%$ were unaware of Junior MARSIPAN. Red flag features were not consistently recognised. However, all could give a basic description of refeeding syndrome. Challenges experienced included adequate nursing supervision, and multidisciplinary team working.

Conclusions Together these reviews highlight the need for improved education amongst paediatricians, including signposting to Junior MARSIPAN. The challenges of effective multi-disciplinary working are described in all. Improved education within schools and primary care is also suggested, facilitating earlier recognition and referral.

\section{G150(P) ABSTRACT WITHDRAWN}

\section{G151(P) IDENTIFYING THE MISSING COHORT: YOUNG PEOPLE WITH COMPLEX NEURO-DISABILITY WHO ARE DIFFICULT TO TRANSITION TO ADULT SERVICES}

\footnotetext{
1,2LA Brook, ${ }^{3}$ J Rogers. 'Specialist Palliative Care Team, Alder Hey Children's Hospital, Liverpool, UK; ${ }^{2}$ International Observatory on Palliative Care, Lancaster University, Lancaster, UK; ${ }^{3}$ Transition Nurse, Alder Hey Children's Hospital, Liverpool, UK
}

\subsection{6/archdischild-2015-308599.147}

Background Young people with complex long term conditions, severe learning disability, technology dependence and palliative care needs present specific additional challenges, making them difficult to transition to adult services. This cohort lacks a single unifying diagnosis making them difficult to identify.
Aims To develop a reliable, reproducible method to identify young people of transition age with complex neurodisability Method Lists of young people aged 14 or over from community physiotherapy, special school, specialist and palliative care clinics were merged. Antenatal mothers, patients who had not attended in the last two years or who had died were removed. The cohort was validated by community children's nursing teams.

Patients were identified using the criteria:

- Severe or profound and multiple learning disability

- 3 or more specialist services

- Technology dependent

- Life threatening or life limiting condition

- Gross motor function measure 4 or 5

- Oxygen dependent

Results 56 young people were identified. Accurate information on learning disability, physical disability, technology dependence and oxygen use was inconsistently recorded in clinic letters. The majority of young people aged 18 or over but none aged under 16 had evidence of transition discussions. Several young people had clinic documentation indicating professional reluctance to transition to adult services because of a lack of appropriate services in the adult sector or a specific application to allow the young person to continue to access childrens services as there was no appropriate service in the adult sector.

$20(36 \%)$ were young people aged 18 years or older who were "stuck" or "delayed" in children's services due to a lack of suitable target services in the adult sector. Approximately half of this group were oxygen dependent.

Conclusion Young people of transition age with complex neurodisability can be identified from routine hospital data. However the required information is not consistently recorded in any one place and the absence of information cannot be equated with the absence of need.

Transition to adult services for this cohort of patients is only being actively considered when the young person is 17 or 18 in contrast to other patients where a recognised transition pathway is in place (e.g. neuromuscular patients) where there was clear evidence of transition discussions from age 14 .

\section{G152(P) ADOLESCENT FEMALES WITH ABDOMINAL PAIN: WHAT INFLUENCES SEXUAL HISTORY TAKING AND PREGNANCY TESTING IN A\&E?}

K Orf, C McDonald, B Sagoo, A Pal. Ealing Hospital, London, UK

\subsection{6/archdischild-2015-308599.148}

The 'Saving Mothers' Lives' report found that between 2006-8 ectopic pregnancies accounted for over $1 \%$ of pregnancies in the UK, and $6 \%$ of maternal deaths. The accident and emergency (A\&E) department is the first port of call for many women with symptoms of abdominal pain, collapse or vaginal bleeding. NICE recommends assessment of any women of a reproductive age must include consideration into whether symptoms are due to an ectopic pregnancy. We performed a six-month retrospective review of adolescents attending the $\mathrm{A} \& \mathrm{E}$ department with abdominal pain and examined factors influencing sexual history taking and urine pregnancy testing.

From April to September 2014, A\&E notes of all female patients aged 13-16 years old presenting with abdominal pain were collated and screened using the computer system Symphony. Fifty patients fitted the selection criteria. The assessing doctor's department and grade was noted and information 